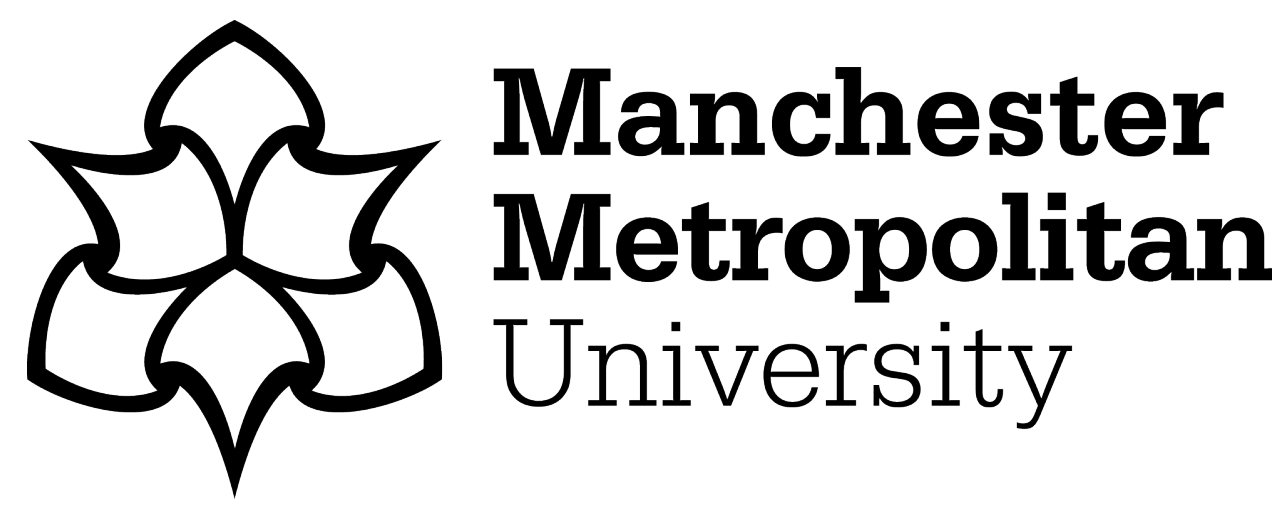

Werner, KM, Amir, R and Gama, A (2018) On Environmental Regulation of Oligopolies: Emission versus Performance Standards. Environmental and Resource Economics, 70 (1). pp. 147-167. ISSN 1573-1502

Downloaded from: https://e-space.mmu.ac.uk/617934/

Version: Accepted Version

Publisher: Springer Verlag (Germany)

DOI: https://doi.org/10.1007/s10640-017-0114-y

Please cite the published version 


\title{
On environmental regulation of oligopoly markets: Emission versus performance standards *
}

Dec 2016

\begin{abstract}
By specializing Montero's (2002) model to an oligopoly with linear demand and quadratic abatement costs, we extend his comparison of the incentives to invest in R\&D under emission and performance standards by solving for a closed form solution of the underlying two-stage game. This allows for a full comparison of the two instruments in terms of their resulting propensity for R\&D and equilibrium industry output. In addition, we incorporate an equilibrium welfare analysis. Finally, we investigate a three-stage game wherein a regulator sets a socially optimal emission cap under each policy instrument. For the latter game, while closed-form solutions are not possible, we establish that the resulting welfare is always larger under a performance standard.
\end{abstract}

${ }^{*}$ We thank two referees of this journal and Hassan Benchekroun (as associate editor) for thorough and thoughtful reports, with many useful suggestions. We also gratefully acknowledge the wonderful hospitality at the Hausdorff Institute for Mathematics, University of Bonn, where this work was initiated, in the summer 2013. 


\section{Introduction}

Due to the increasing importance of environmental issues, a large body of theoretical work has emerged in recent years to examine the performance of environmental policy instruments by means of the incentives these instruments provide to invest in R\&D. Requate (2005) offers a detailed survey of studies investigating the performance of the usual regulatory instruments in various market structures allowing for market power in the hands of polluting firms. ${ }^{1}$ The study of particular interest to us is Montero (2002), who considers a basic and very general model of a two-stage game under four different regulatory regimes. The basic two-stage game entails Cournot firms that decide on R\&D levels for improved abatement technology in the first period, and then compete in Cournot outputs in the second stage. The four regulatory regimes under which firms operate consist of two command-and-control policy instruments, emission and performance standards, and two market-based instruments. With all four policy instruments exogenously set, Montero's analysis concludes that, due to the presence of strategic effects, the command-and-control instruments may offer greater incentives than market based instruments, contrary to what had been claimed previously.

Montero's reliance on a general model of a Cournot market carries the usual benefits of robustness of conclusions and elegance of the underlying analysis. However, in the present case, coupled with the perhaps overly ambitious goal of comparing four different instruments, it also leads to the impossibility of a fully-fledged comparison, and thus to the abscence of clear-cut answers to some desirable questions. The main purpose of the present paper is to consider two of Montero's models under a standard oligopoly specification that allows for an elementary, more detailed, and in-depth analysis, and thus provides clear-cut answers to some of the interesting comparisons left out by Montero (more on this below). ${ }^{2}$ We focus on the models with the emission and performance standards, in order to fully analyze the R\&D incentives that these two policy instruments offer, in a comparative perspective.

Though clearly a central question in environmental economics, the comparison between stan-

\footnotetext{
${ }^{1}$ A very partial literature listing would include the following studies: Downing and White, 1986; Jung, Krutilla and Boyd, 1996; Malueg, 1989, Milliman and Prince, 1989; Benchekroun and Van Long, 1998, and many others.

${ }^{2}$ Importantly, the questions of existence and uniqueness of a subgame-perfect equilibrium in pure strategies for the four different games analysed by Montero (2002) have not been addressed, and probably constitute very challenging issues in themselves. These issues can end up being crucial for the well-foundedness of the models at hand, e.g., for some of the properties of equilibria, including their comparative statics properties.
} 
dards and market-based instruments is beyond the scope of the present paper. There are several different motivations behind this choice. The first is real-world relevance: For better of for worse, the command-and-control regime is known to be the dominant policy approach to environmental regulatory design (see e.g., Hueth and Melkonyan, 2009). ${ }^{3}$ The second is of a more practical nature, reflecting a simple wish to avoid an overly lengthy, heterogeneous, and unfocused paper. Importantly, the structure of the two games corresponding to the two standards is quite similar, but very different from the structure of the games corresponding to the two market-based instruments. ${ }^{4}$

As in Montero (2002), the specific abatement technology we consider throughout the paper is end of pipe abatement. ${ }^{5}$ The contribution of the present paper to the analysis of the R\&D incentives generated by the regulatory standards is two-fold. First, the present paper focuses on a standard specification of Cournot oligopoly, with linear demand, production and abatement costs. By adopting specific functional forms, we forsake generality but can derive some relevant fully-fledged equilibrium properties and more transparent comparison results for the two-stage games. In particular, we provide a complete characterization of the comparison between the propensities for R\&D and for higher output generated by each of the two policy instruments. This enables us to fully assess the market features under which each instrument is superior in terms of generating more R\&D investment and more output. In contrast, Montero considers a Cournot framework that is so general that one cannot conduct a full-fledged equilibrium analysis of the underlying two-stage games. Thus, in particular one cannot infer the extent to which the incentives to invest in $\mathrm{R} \& \mathrm{D}$ vary with the parameters of interest (such as the level of demand, the emissions ceiling and the $R \& D$ cost parameter). Instead, Montero uses as a criterion for

\footnotetext{
${ }^{3}$ For further and more detailed discussion of the fact that the regulation of environmental risk is dominated by the use of standards, the reader is referred to Viscusi et al. (2000) and Harrington et al. (2004). Nevertheless, this state of affairs often does not reflect economists' views on the normative merits of the two types of instruments.

${ }^{4}$ One implication of this fact is that, even with our simple specification, a four-way comparison appears possible only via numerical simulations. The difference in structure across the two types of games also raises some issues as to the very meaning of some of the comparisons.Finally, the difference in model structure also means that the appropriate functional forms might not be the same.

${ }^{5}$ There are multiple ways of modeling R\&D in an environmental setting (see e.g., Amir et al., 2008). The main alternative to end of pipe technology is the use of cleaner technologies of production, which lead to a lower emissions-output ratio. Whether the conclusions of the present paper extend to this $\mathrm{R} \& \mathrm{D}$ technology is an interesting topic for future work, but beyond the scope of this paper.
} 
comparison firms' incentives to invest in $R \& D$ as measured by its marginal payoff to $R \& D$ in the two-stage game, conditional on a second-stage Cournot equilibrium.

To describe the second contribution of this paper, recall that Montero (2002) does not rank the regulatory instruments with respect to total welfare. In his paper, the planner enforces exogenously given, and mutually consistent, aggregate pollution constraints for the two scenarios. In this regard, the present paper extends Montero's analysis in two separate but related extensions. We first compare the levels of equilibrium welfare generated by the two policy instruments in the two-stage game. In a more substantive modification, we endogenize the regulatory standards in a three-stage game via the inclusion of the social planner as a player in an additional stage at time 0 , with the other two stages remaining as before. The objective of the social planner is social welfare maximization.

Taking into account the objective of the regulator enables a full equilibrium analysis, hence, a comparison of the actual welfare implications of firms' adoption of new abatement technologies under imperfect competition. As argued by Requate (2005), incorporating welfare in the equilibrium analysis is important as it may lead to quite different results regarding the performance of the regulatory instruments. Thus, by providing such an analysis within the aforementioned specification, the present paper can be viewed as complementary to Montero's work. As can be easily expected, the three-stage game at hand would not be tractable under Montero's broad level of generality. ${ }^{6}$

We now preview the results of the present papers. We start with the solution to the two-stage games, for which the emission targets are exogenously set. Confirming the results of Montero (2002), we show that the levels of R\&D investment and output generated under each policy instrument depend on the underlying market and R\&D parameters. Going beyond Montero, however, we use the simple specification of our model to provide a complete description of parameter regions for which each instrument is superior to the other in terms of generating more R\&D investment and more industry output, respectively. We find that neither of the two command-and-control instruments is uniformly superior in providing more incentives for $\mathrm{R} \& \mathrm{D}$ in abatement technology or for more industry output. Moreover, we compare the resulting welfare levels under the two policy instruments and demonstrate via specific numerical examples that

\footnotetext{
${ }^{6}$ Even for our simple specification, the basic three-stage model is not as analytically tractable as the two-stage games we investigate.
} 
this comparison can go either way.

In contrast, for the three-stage game in which the regulator's objective is to maximize social welfare, we establish that the performance standard is welfare superior to the emission standard. This conclusion is consistent with real-life observation, in the sense that the performance instrument is commonly chosen by regulators (Bruneau, 2004, p. 1194; Requate, 2005, p.178). In light of this positive and clear-cut result, we conclude by emphasizing the importance that endogenizing the regulatory standard via a social welfare objective has for the optimal choice of environmental policy instruments (Requate, 2005).

In terms of the limitations of our analysis, it is clear that the reliance on the standard specification of a linear oligopoly raises the usual issues of potential non-robustness of the conclusions to other plausible specifications. Nevertheless, this specific setting is intended as a complement to Montero's (2002) general approach. As noted earlier, due the the usual complexities associated with strategic behavior, the latter approach has yielded limited insights precisely because of its level of generality.

The rest of the paper is organized as follows. The next section sets out the basic model. Section 3 provides the analysis of the two-stage game under the emission and performance standards. The extension to the three-stage game is given in Section 4. Section 5 concludes. All proofs are given in the Appendix.

\section{Preliminaries of the model}

Consider a duopoly market with three agents: two ex-ante symmetric profit maximizing firms and a regulator. In the absence of any environmental regulation, firm $i$ produces quantity $q_{i}$ at no cost, so that the aggregate quantity produced in the market equals $Q=q_{i}+q_{j}$, for $i, j \in\{1,2\}, i \neq j$, and the (inverse) demand function is given by $P(Q)=a-b Q$, with $a, b>0 .{ }^{7}$ Since production of firm $i$ leads to $q_{i}$ units of emissions, which cause damage to the environment, firm $i$ becomes subject to environmental regulation.

To reduce the level of pollution in the environment, the regulator has a choice of one of two

\footnotetext{
${ }^{7}$ In analogy to Montero (2002), we abstract away from the production cost to concentrate solely on the effects of new abatement technology on abatement cost. The impact of increasing marginal cost on the R\&D incentives has been considered by Bruneau (2004), who, however, investigates this impact only in perfectly competitive output markets.
} 
command-and-control policy instruments: Emission or performance standards. The emission standard constrains the level of pollution generated by firm $i$ to be under an upper limit $e_{i}$, while the performance standard imposes a restriction on firm $i^{\prime}$ s maximal ratio of emissions per output, $h_{i}=e_{i} / q_{i}$. The aim of the regulator is to restrict the total emissions generated in the market, $E=e_{i}+e_{j}$, and, thereby, to reduce the resulting environmental damage. The damage is posited to be given by $D(E)=s E^{2} / 2$, where $s>0$, a common and convenient specification reflecting the fact that per-unit damage increases with the emission level.

Whenever firm $i$ exceeds the pollution standard imposed by the regulator, the firm is under the obligation to abate the excess emissions and incur the corresponding abatement cost. The latter is given by a function $C\left(y_{i}, x_{i}\right)=\left(c-x_{i}\right) y_{i}$ for firm $i$, where $c>0$ is the constant marginal abatement cost, $y_{i}$ denotes the amount of emissions abated, and $x_{i}$ denotes investment in R\&D. This simple specification reflects the general feature that the abatement cost function of firm $i$ increases in the amount of emissions abated, given by $y_{i}=q_{i}-e_{i}$ for the emission standard, and by $y_{i}=q_{i}\left(1-h_{i}\right)$ for the performance standard, with $h_{i} \in(0,1)^{8}$. In addition, the R\&D investment, $x_{i}$, improves the abatement technology of firm $i$ by reducing its unit abatement cost.

To describe the $R \& D$ process, we assume that the $R \& D$ technology is deterministic and reflects diminishing returns to $R \& D$ expenditures. For convenience, we postulate that the cost of reducing the constant marginal abatement cost by $x_{i} \geq 0$ follows a quadratic function, namely $g\left(x_{i}\right)=\gamma x_{i}^{2} / 2$, where $\gamma>0 .^{9}$

For polluting firms, there are several different ways of modeling R\&D in abatement technology. Implicit in this description of environmental $R \& D$ is the fact that that the firms reduce their emissions by undertaking end-of-pipe abatement. Indeed, the objective of environmental $\mathrm{R} \& \mathrm{D}$ here is to reduce the cost of pollution abatement. ${ }^{10}$

\footnotetext{
${ }^{8}$ We do not consider the extreme cases, in which $h_{i}=0$ and $h_{i}=1$, since they imply that firm $i$ does not emit, or $y_{i}=q_{i}$, and that it does not abate, or $y_{i}=0$, respectively.

${ }^{9}$ Due to its tractability, the quadratic R\&D cost specification is quite standard (e.g., d'Aspremont and Jacquemin, 1988 and Amir, 2000).

${ }^{10}$ Another common alternative way to introduce environmental R\&D is to allow firms to use cleaner technologies to comply with environmental regulation. In this case, environmental R\&D would aim at reducing the ratio of emissions per output. Whether the main results of the present paper extend to this type of environmental R\&D is an open question of substantial interest. Recent results on some hitherto unknown differences between the two types of R\&D indicate that the answer to this question requires some substantive analysis (see Amir et al, 2008, Baumann et al., 2008 and Baker et al., 2008 and Brechet- Jouvet, 2008, for more on this important point).
} 
For each of the two types of pollution constraint imposed by the regulator, the two-stage game under consideration unfolds as follows. In the first stage, the two firms simultaneously choose their environmental R\&D investments. In the second stage, they compete by choosing output levels (à la Cournot) upon observing the two first stage R\&D decisions.

\section{The two-stage game}

It is common in the literature on the merits of environmental policy instruments to postulate that the emission constraint is exogenous, implying that the regulator is not an active part of the model. In addition, due to the lack of tractability of his general formulation, Montero (2002) does not base the performance comparisons on equilibrium levels of $R \& D$ in the four different games, but on the marginal profit to $R \& D$ for each fixed possible level of $R \& D$. This pointwise comparison criterion might be too demanding, and the ambiguity in the resulting comparisons might well be due to the stringency of this criterion. With the goal of obtaining more clear-cut comparisons, we relax this criterion to compare only the equilibrium R\&D values, which clearly form the only relevant levels for each model.

To this end, we exploit the tractability of our simple formulation and solve for the equilibrium of the two-stage game via backwards induction. Thus, we assume that firms correctly anticipate the equilibrium level of output, when deciding on their level of R\&D investment to maximize profit. Moreover, we make the following assumption:

$$
\text { (A1): } 9 b \gamma>8,9 b \gamma>4 a / c, a>2 c .
$$

These parameter restrictions ensure that the solutions to the two-stage games are economically meaningful, yielding positive levels of abatement cost and output and ensuring that the level of emissions is below that of output. ${ }^{11}$

\subsection{The emission standard}

In this model, the regulator imposes a cap on the level of emissions that a firm can generate via its production. Since the firms are symmetric, we assume that the regulator sets the same emission ceiling, $e$, for both firms. In the second stage of the game, firm $i$ chooses its own

\footnotetext{
${ }^{11}$ In addition, this assumption gets rid of potential technical difficulties and long computations (that a priori have no particular economic content of interest) associated with boundary equilibrium solutions.
} 
output, $q_{i}$, and the final level of emissions (after abatement), $e_{i}$, to maximize its profit given the levels of $e$, its R\&D investment $x_{i} \in[0, c]$, and its rival's output, $q_{j}$ (recall that $c>0$ is the unit abatement cost). Thus, the total emissions abated by the firm equal $q_{i}-e_{i}$. Hence, the optimization problem of firm $i$ in this stage is given by

$$
\max _{q_{i}, e_{i}} q_{i}\left[a-b\left(q_{i}+q_{j}\right)\right]-\left(c-x_{i}\right)\left(q_{i}-e_{i}\right) \text { s.t. } e_{i} \leq e .
$$

Since the objective function in (1) is increasing in $e_{i}, e_{i}=e$ is set, and the profit-maximization problem reduces to

$$
\max _{q_{i}} q_{i}\left[a-b\left(q_{i}+q_{j}\right)\right]-\left(c-x_{i}\right)\left(q_{i}-e\right),
$$

which leads to equilibrium individual outputs $q_{i}\left(x_{i}, x_{j}, e\right)$, for $i, j \in\{1,2\}, i \neq j$. ${ }^{12}$

In the first stage, the firms simultaneously choose their R\&D investments, $x_{i}$ and $x_{j}$, given $e$ and the equilibrium in the second stage. Thus, firm $i$ solves the following problem

$$
\max _{x_{i}} q_{i}\left(x_{i}, x_{j}, e\right)\left[a-b\left(q_{i}\left(x_{i}, x_{j}, e\right)+q_{j}\left(x_{i}, x_{j}, e\right)\right)\right]-\left(c-x_{i}\right)\left[q_{i}\left(x_{i}, x_{j}, e\right)-e\right]-\frac{\gamma x_{i}^{2}}{2} .
$$

The last term in (3) denotes the cost that firm $i$ incurs when investing the amount of $x_{i}$ in R\&D.

Due to the symmetry of the game, this stage leads to a symmetric equilibrium, fully described by the level of R\&D investment, $x(e)$, and output, $q(e)$. For this equilibrium, we require that $0<e<\frac{3 \gamma(a-c)}{9 b \gamma-1}$, to ensure that our results are economically meaningful (i.e., this assumption ensures that the levels of abatement cost and output are positive, and that the level of emissions is below that of output).

Lemma 1 Under assumption (A1), there is a unique symmetric subgame-perfect equilibrium of the two-stage game under the emission standard, with equilibrium RESD and outputs levels respectively given by

$$
x(e)=\frac{4(a-c)-9 b e}{9 b \gamma-4} \text { and } q(e)=\frac{3 \gamma(a-c)-3 e}{9 b \gamma-4} .
$$

Moreover, $0<x(e)<c$ and $0<e<q(e)$.

The next section provides a solution to the two-stage game under the performance standard.

\footnotetext{
${ }^{12}$ The assumptions of this model imply that equilibrium does not depend on $e$ in this stage, hence, we have $q_{i}\left(x_{i}, x_{j}\right)$ for $i, j \in\{1,2\}$ and $i \neq j$.
} 


\subsection{The performance standard}

A performance standard refers to a cap, $h$, which the regulator imposes on the proportion of pollution that a firm can emit per unit of production. For instance, if the regulator chooses $h=0.7$, the firm's emissions can at most amount to $70 \%$ of its output. Hence, if $q=100$, emissions must be reduced to 70 , so that $q(1-h)=30$ units of emissions must be abated.

Given a choice of $h$ by the regulator, $x_{i}$ from the first stage, and rival output $q_{j}$, the profitmaximizing firm $i$ solves in the second stage the following problem:

$$
\max _{q_{i}} q_{i}\left[a-b\left(q_{i}+q_{j}\right)\right]-\left(c-x_{i}\right) q_{i}(1-h),
$$

where the equilibrium output in this stage is denoted by $q_{i}\left(x_{i}, x_{j}, h\right)$ for firm $i$.

In the first stage, the objective of firm $i$ is given by

$$
\max _{x_{i}} q_{i}\left(x_{i}, x_{j}, h\right)\left[a-b\left(q_{i}\left(x_{i}, x_{j}, h\right)+q_{j}\left(x_{i}, x_{j}, h\right)\right)\right]-\left(c-x_{i}\right) q_{i}\left(x_{i}, x_{j}, h\right)(1-h)-\frac{\gamma x_{i}^{2}}{2},
$$

where the last term in equation (5) denotes the cost of R\&D.

Due to the symmetry of the game, we obtain a (unique) symmetric subgame-perfect equilibrium. Denote the equilibrium variables by $x(h)$ and $q(h)$.

Lemma 2 Assume (A1) holds. Then, for all $0<h<1$, there is a unique symmetric subgameperfect equilibrium of the two-stage game under the performance standard, with REDD and output levels respectively given by

$$
x(h)=\frac{4(1-h)[a-c(1-h)]}{9 b \gamma-4(1-h)^{2}} \text { and } q(h)=\frac{3 \gamma[a-c(1-h)]}{9 b \gamma-4(1-h)^{2}} .
$$

Moreover, $0<x(h)<c$ and $0<q(h)$.

The next section compares the solutions to the two-stage games under the two policy instruments.

\subsection{Emission versus performance standards in the two-stage game}

The aim of this section is to compare the emission and performance standards for the twostage game formulation in terms of the equilibrium incentives to invest in environmental R\&D, and to produce a higher output. In addition, the comparison is extended to the resulting social welfare. 
As a way to provide an intuitive account of the forces at work in the two models, Montero (2002) assesses the incentives to invest in environmental R\&D by means of two effects: the direct (cost-minimizing) effect and the strategic effect. The direct effect refers to the savings associated with the adoption of a new abatement technology, and is increasing in the amount of abatement. The strategic effect accounts for the fact that one firm's R\&D investment lowers the rival's output, market share and profit, through the usual business-stealing effect of Cournot competition.

Montero (2002) finds that the direct effect is larger under the emission standard, while the strategic effect is larger under the performance standard. As the investment in R\&D increases, the abatement cost decreases, leading to a rise in the level of output. According to Montero, this leads to an increase in the amount of emissions that are allowed under the performance standard. Under the emission standard, the pollution constraint is unaffected by the change in output. Thus, a higher level of emissions must be abated under an inflexible emission standard, which requires a higher abatement cost. This gives a firm a greater incentive to invest more in $R \& D$ under the emission standard, where greater savings from abatement are anticipated. On the other hand, the increase in the level of output induced by the lower cost of abatement enables a firm to achieve a larger market share and profit under the performance standard. Consequently, as the two effects move in opposite directions, the outcome of Montero's comparison of the R\&D incentives under the emission and performance standards is ambiguous.

Exploiting the simple tractable formulation of the present paper, the main result of this section adds to Montero's (2002) findings by providing a complete characterization of the comparison between the propensities for higher R\&D and output generated by each of the two policy instruments. A welfare comparison then follows.

Proposition 3 contains a complete description of the parameter regions (including the values of $h$ ) for which each instrument is superior to the other, in terms of generating more R\&D investment and more output, respectively.

To ensure a meaningful comparison of the incentives to invest in R\&D under both regulatory instruments, we assume that the regulator chooses the emission constraints $e$ and $h$ so that the final level of emissions is the same for the two instruments. In other words, if $q(h)$ is the equilibrium output produced under the performance standard in the two-stage game, $e$ is chosen 
so that $e=q(h) h \cdot{ }^{13}$ Define the thresholds $\bar{h} \triangleq \frac{5(9 b \gamma)-(9 b \gamma+16) a / c}{9 b \gamma-16 a / c}$ and $h_{0} \triangleq \frac{5 a-9 b c \gamma-c}{4 a-c} .{ }^{14}$

Proposition 3 Under Assumption (A1), the RED/output comparison of the two standards is:

a) Let $a / c<9 b \gamma / 16$. Then,

(i) if a/c $<4$, a performance standard leads to more R\&D.

(ii) if $a / c>5 \frac{9 b \gamma}{9 b \gamma+16}$, an emission standard leads to more R\&D.

(iii) If $4<a / c<5 \frac{9 b \gamma}{9 b \gamma+16}$ and $h<(>) \bar{h}$, a performance (emission) standard leads to more RGBD.

b) Let $a / c>9 b \gamma / 16$. Then,

(i) if a/c $>4$, an emission standard leads to more REDD.

(ii) if $a / c<5 \frac{9 b \gamma}{9 b \gamma+16}$, a performance standard leads to more $R \mathscr{E} D$.

(iii) If $5 \frac{9 b \gamma}{9 b \gamma+16}<a / c<4$ and $h<(>) \bar{h}$, an emission (performance) standard leads to more $R E D$.

c) (i) If $a / c<(9 b \gamma+1) / 5$, the performance standard leads to more output.

(ii) If $a / c>(9 b \gamma+1) / 5$ and $h<(>) h_{0}$, an emission (performance) standard gives more output.

Proposition 3 provides a complete characterization of the comparison between the propensities for abatement R\&D and for industry output that are generated by the two standards. The comparison turns out to be quite complex, with neither of the two instruments emerging as uniformly superior. Instead, the comparison is highly parameter-dependent, with the following conclusions nevertheless essentially holding. To state these, we shall take $a / c$ as being a measure of market size (for fixed initial unit cost $c$ ), and $\gamma$ as being a measure of the cost of abatement R\&D. ${ }^{15}$

Considering parts (a) and (b) together, the main message is as follows. When R\&D is sufficiently costly (at fixed market size), a performance standard generates more R\&D. On the other hand, when market size is sufficiently large (at fixed unit cost of R\&D), an emission standard generates more R\&D. For modest levels of market size and $R \& D$ cost, the outcome of the comparison is less clear-cut, although similar forces appear to be at work here too.

Considering part (c), the effects on equilibrium industry output are qualitatively similar to those on $R \& D$. A large market size favors an emission standard, while costly abatement R\&D

\footnotetext{
${ }^{13}$ As in Proposition 1, we additionally require that $0<e<\frac{3 \gamma(a-c)}{9 b \gamma-1}$, to ensure that $0<x(e)<c$ and $0<e<q(e)$.

${ }^{14}$ Under the conditions of Proposition 3 (a)(iii) and (b)(iii), it is useful to note that $0<\bar{h}<1$.

${ }^{15} \mathrm{~A}$ convenient feature of the quadratic cost function is that firms will always choose to undertake striclty positive levels of $R \& D$ even when $\gamma$ is very high.
} 
favors a performance standard.

The following example is a simple illustration of the results of Proposition 3.

Example 1: Suppose that $b=c=1$, and $a=2.5$. Table 1 gives the threshold values of the performance standard for which Proposition 3 holds. These levels are given by $\bar{h}$ and $h_{c}$, and are computed for some feasible values of the parameter denoting a unit cost of R\&D, $\gamma$.

\begin{tabular}{lcccccc}
\hline Variable & $\begin{array}{c}h_{a b} \\
(\gamma=1.15)\end{array}$ & $h_{c}$ & $h_{a b}$ & $h_{c}$ & $h_{a b}$ & $h_{c}$ \\
& & $(\gamma=1.20)$ & & $(\gamma=1.80)$ & \\
\hline Threshold & 0.48 & 0.13 & 0.45 & 0.08 & -0.02 & -0.52 \\
\hline
\end{tabular}

Table 1: Thresholds in Proposition 3 for $b=c=1, a=2.5$ and different values of $\gamma$.

Here, $\gamma$ has been chosen so that $9 b c \gamma-16 a<0$ holds (i.e., case (b) of Proposition 3 applies). Thus, Table 1 shows that for a low cost of $\mathrm{R} \& \mathrm{D}(\gamma=1.15)$, more $\mathrm{R} \& \mathrm{D}$ is undertaken and more output is produced under the emission standard if $0<h<0.48$ or if $0<h<0.13$, respectively. The case with $\gamma=1.20$ can be interpreted similarly.

For $\gamma=1.80$, the interpretation is as follows. Since both thresholds are negative, $h_{a b}=-0.02$ and $h_{c}=-0.52$, the second condition on $h$ cannot hold in Proposition 3 (a)-(c), since $h$ is always $>0$. Then Proposition 3 (a)-(b) says that the performance standard always generates a lower (resp., higher) investment in $\mathrm{R} \& \mathrm{D}$ if $9 b c \gamma-16 a>0$ (resp., if $9 b c \gamma-16 a>0$ ). Part (c) says that the performance standard yields a higher output.

An analogous analysis can be undertaken for changes in other parameters of interest.

Proposition 3 clarifies that neither of the two command-and-control instruments is (uniformly) superior in generating the incentives to invest in environmental $\mathrm{R} \& \mathrm{D}$ or to produce more output. For each of the latter criteria, there

In light of this ambiguous outcome, a natural question to ask is whether comparing the resulting social welfare levels always leads to a clear-cut conclusion. Assuming that $e=q(h) h$ and defining equilibrium welfare as

$$
\int_{0}^{2 q(h)} P(t) d t-2[c-x(h)] q(h)(1-h)-\frac{s(2 h q(h))^{2}}{2}-\gamma[x(h)]^{2}
$$


we find that the answer to this question is negative, as demonstrated by means of the following counterexample.

Example 2: Consider our two-stage game formulation with the values of parameters $b, c, s, \gamma$, and $a$ being fixed, so that $b=c=s=1, \gamma=1.8$, and $a=1.2$. The resulting values of equilibrium welfare together with $R \& D$ investment, output, and final emissions per firm under both policy instruments are shown in Table 2 , where $h=0.1$ and $h=0.3$.

\begin{tabular}{lcccl}
\hline Variable & e.s. & p.s. & e.s. & p.s. \\
& $h=0.10$ & & $h=0.30$ & \\
\hline R\&D per firm & 0.056 & 0.083 & 0.024 & 0.098 \\
Individual output & 0.085 & 0.125 & 0.075 & 0.190 \\
Welfare & 0.047 & 0.050 & 0.126 & 0.120 \\
Final emissions per firm & 0.013 & 0.013 & 0.057 & 0.057 \\
\hline
\end{tabular}

Table 2: Variables of interest for different values of $h$ and $b=c=s=1, \gamma=1.8 a=1.2$.

The choice of $h=0.1$ and $h=0.3$ in Table 2 is not random. In particular, for $h=0.1$, we demonstrate that the performance standard generates a higher level of welfare than the emission standard. ${ }^{16}$ However, for $h=0.3$, we are able to show the opposite - the emission standard is now superior in generating a higher level of welfare. ${ }^{17}$ Thus, this counterexample demonstrates that the welfare comparison between the two instruments depends critically on the values of parameter $h$.

As to welfare, unfortunately, a complete characterization of parameter regions for which one instrument is welfare superior to the other (in the spirit of Proposition 3 for R\&D and output levels) does not appear tractable, even for our convenient quadratic formulation.

The absence of a clear-cut conclusion for the welfare comparison in the two-stage game provides a further motivation to investigate a three-stage game scenario, in which social welfare

\footnotetext{
${ }^{16}$ At $h=0$, welfare under the emission and performance standards coincides, but the derivative of the welfare function with respect to $h$ is higher under the performance standard. In particular, one obtaines $W^{p \prime}(0)-$ $W^{e \prime}(0)=\frac{2(a-c) \gamma(9 b c \gamma-4 a)}{(4-9 b \gamma)^{2}}>0$, where $W^{p \prime}(0)$ and $W^{e \prime}(0)$ denote the derivatives of the welfare function under the performance and emission standards, respectively.

${ }^{17}$ We find that the performance standard generates higher welfare than the emission standard for $h<0.22$.
} 
is endogenously determined. To this end, we integrate the regulator as an active player in the game and postulate social welfare as his objective function. The next section analyzes this scenario.

\section{The three-stage game}

In contrast to the two-stage game, in which emission and performance standards were set in an exogenous manner, this section analyzes the socially optimal pollution levels arising under these policy instruments. Thus, the game under consideration now comprises three instead of two stages. Under each policy instrument, the additional stage (at time 0) allows the regulator to select the level of emissions that maximizes social welfare before the firms make their sequential decisions about their levels of $R \& D$ investment and their outputs. Hence, the pollution standards are thereby determined endogenously. The regulator sets the pollution level by taking into account the environmental damage that this level will be associated with, thus anticipating firms' equilibrium reactions. The remaining two stages (at time 1 and 2) are the same as those considered in the two-stage game: the first stage involves firms simultaneously choosing their R\&D investments, while the second stage is the Cournot competition phase.

For this three-stage game we shall require throughout that, in addition to assumption (A1), the following assumption holds:

(A2): $9 b+8 s<18 b \gamma s, a<2 \gamma s(a-c), 9 b c<8 s(a-c), 9 b c \gamma-6 \gamma s(a-c)<a$.

This assumption ensures that the solutions to the three-stage games are economically meaningful, yielding positive levels of abatement cost and output and ensuring that the level of emissions is below that of output. ${ }^{18}$

\subsection{Emission standard}

Recall that the two-stage equilibrium values of $\mathrm{R} \& \mathrm{D}$ investment, $x(e)$, and output, $q(e)$, depend on the emission target, $e$, which was set exogenously by the regulator. In contrast, by pursuing the objective of social welfare maximization, the regulator derives the emission cap

\footnotetext{
${ }^{18}$ Notice that $a<2 \gamma s(a-c)$ in (A2) implies $a>c$ in $(\mathbf{A 1})$, for the positive values of the parameters. Yet, we list $a>c$ as a separate assumption because this weaker version is sufficient for some proofs (see the Appendix).
} 
endogenously in the three-stage game. In particular, he solves the following welfare maximization problem:

$$
\max _{e} \int_{0}^{2 q(e)} P(t) d t-2[c-x(e)][q(e)-e]-\frac{s(2 e)^{2}}{2}-\gamma[x(e)]^{2},
$$

where the industry emissions are equal to $E=2 e$.

The first term in equation (6) corresponds to the consumer surplus, while the second term

reflects the costs of abatement incurred by both firms. The remaining expressions, $\frac{s(2 e)^{2}}{2}$ and $\gamma[x(e)]^{2}$, capture the damage and the total cost of $\mathrm{R} \& \mathrm{D}$, respectively.

Given the two-stage equilibrium values of R\&D investment, $x(e)$, and output, $q(e)$ (recall Lemma 1), the regulator's problem reduces to

$$
\max _{e}\left\{\frac{e^{2}(9 b+8 s-18 b \gamma s)+e(18 b c \gamma-8 a)+4 \gamma(a-c)^{2}}{9 b \gamma-4}\right\} .
$$

The solution to this problem is given by Lemma 4, in which the variables of interest carry an additional index, $(\cdot)^{*}$, to distinguish this solution from the solution to the two-stage game.

Lemma 4 Under assumptions $(\boldsymbol{A 1})$-(A2), there is a unique symmetric equilibrium for the three-stage game under the emission standard, which entails the social welfare maximizing level of emission constraint, REDD levels, and outputs respectively given by

$$
e^{*}=\frac{4 a-9 b c \gamma}{9 b+8 s-18 b \gamma s}, x^{*}=\frac{9 b c-8 s(a-c)}{9 b+8 s-18 b \gamma s}, \text { and } q^{*}=\frac{3 a-6 \gamma s(a-c)}{9 b+8 s-18 b \gamma s} .
$$

Moreover, $0<x^{*}<c$ and $0<e^{*}<q^{*}$.

The next section provides a solution to the three-stage game under the performance standard.

\subsection{Performance standard}

In the three-stage game, the regulator chooses $0<h<1$ at the initial stage (time 0 ) as to maximize social welfare given the equilibrium values of the two-stage game, $x(h)$ and $q(h)$, characterized in Proposition 2. Thus, the regulator solves the following welfare maximization problem

$$
\max _{h} \int_{0}^{2 q(h)} P(t) d t-2[c-x(h)] q(h)(1-h)-\frac{s[2 h q(h)]^{2}}{2}-\gamma[x(h)]^{2} .
$$

Applying results obtained in Proposition 2, the regulator's objective reduces to (upon a long computation)

$$
\max _{h}\left\{\frac{2 \gamma[a-c(1-h)]^{2}\left[9 \gamma\left(2 b-h^{2} s\right)-8(h-1)^{2}\right]}{\left[4(1-h)^{2}-9 b \gamma\right]^{2}}\right\}
$$


Unfortunately, the solution to the regulator's problem is analytically non-tractable. ${ }^{19}$ Hence, we compute the solution for a particular set of parameters. Specifically, we distinguish three cases; case one, in which the value of parameter $a$, the consumers' willingness to pay, varies; case two, in which the value of $\gamma$, the unit cost of $\mathrm{R} \& \mathrm{D}$, varies; and case three, in which the extent of damage, as measured by parameter $s$, varies. For each case, the remaining variables are kept fixed. In so doing, we are able to compare the equilibrium outcomes of the three-stage game under both policy instruments, while maintaining the robustness of the results to the parameter specification. The next section discusses the outcome of the comparison.

\subsection{Comparison of equilibria}

This section compares the equilibria of the three-stage game under both policy instruments in terms of the resulting $R \& D$ levels and outputs. However, due to the non-tractability of the solution to the three-stage game under the performance standard, the comparison is provided for restricted parameter values. In particular, for this section, a simplifying assumption that $b=c=1$ is maintained. Nonetheless, we shall have more to say about the generality of our results below.

The equilibrium variables under the emission standard are given by Proposition 4. Under the performance standard, the solutions and qualitative characteristics are obtained computationally. In both cases, the outcomes of the games are symmetric and carry the superscript $e$ or $p$, for the emission and performance standards, respectively. Below, $W$ denotes total welfare, $e^{*}$ stands for the equilibrium level of pollution under the emission standard, and $h^{*}$ denotes the equilibrium level of ratio of emission to output under the performance standard.

\subsubsection{Varying parameter $a$}

In this section we compare the levels of $\mathrm{R} \& \mathrm{D}$ investment, output and welfare obtained under both policy instruments in order to identify which of the two regimes is superior. Table 3 provides such a comparison for different values of the market size, a. The abbreviation e.s. stands for emission standard, and p.s. for performance standard.

Table 3 demonstrates that an increase in the market size, $a$, encourages firms to produce more.

\footnotetext{
${ }^{19}$ There are four roots in total, however, two of them are imaginary ones and one real root induces zero output. The restant root constitutes as the only relevant solution to the maximization problem of the regulator.
} 


\begin{tabular}{lcccccccc}
\hline Variable & e.s. & p.s. & e.s. & p.s. & e.s. & p.s. & e.s. & p.s. \\
& $(a=2.3)$ & & $(a=2.5)$ & & $(a=3.1)$ & & $(a=3.3)$ & \\
\hline Standard & 0.43 & 0.65 & 0.35 & 0.59 & 0.11 & 0.31 & 0.03 & 0.12 \\
R\&D per firm & 0.14 & 0.21 & 0.30 & 0.27 & 0.78 & 0.57 & 0.94 & 0.82 \\
Individual output & 0.48 & 0.68 & 0.60 & 0.73 & 0.96 & 0.94 & 1.08 & 1.05 \\
Welfare & 1.26 & 1.37 & 1.55 & 1.67 & 2.80 & 2.84 & 3.34 & 3.35 \\
Final emissions per firm & 0.43 & 0.44 & 0.35 & 0.43 & 0.11 & 0.29 & 0.03 & 0.13 \\
Abatement per firm & 0.05 & 0.24 & 0.25 & 0.30 & 0.85 & 0.65 & 1.05 & 0.92 \\
\hline
\end{tabular}

Table 3: Solution to the three-stage game for $b=c=s=1, \gamma=1.5$ and chosen values of $a$.

The increasing level of output, $q$, leads to a higher level of pollution in the environment, which negatively affects social welfare. Thus, the regulator concerned with social welfare maximization makes the pollution constraint more stringent, which is manifested through a drop in the final emissions per firm (the fifth row in Table 3). Since the output increases and the pollution constraint is stricter (lower), the number of abatement units rises (see the sixth row of Table 3). Therefore, the abatement cost rises too, providing firms with more incentives to invest in $\mathrm{R} \& \mathrm{D}$ (the second row in Table 3). Finally, the level of equilibrium welfare increases (the forth row in Table 3).

These results are complemented by Proposition 5, which provides a comparison of the emission and performance standards for all relevant values of parameter $a$. These values are chosen to ensure that our results are economically meaningful. In particular, the lower bound on $a$, $a=2.25$, has been chosen to ensure that under the emission instrument the optimal output level is greater than the emissions level, hence, $q^{e}>e^{*}$. The upper bound of $a, a=3.375$, guarantees that the optimal emission constraints, $e^{*}$ and $h^{*}$, are non-negative. The constants $\gamma=1.5$ and $s=1$ have been chosen so as to lead to a representative outcome.

Proposition 5 Let $b=c=s=1$ and $\gamma=1.5$. Then

a) for $2.25<a<2.433, x^{p}>x^{e}$, and $q^{p}>q^{e}$;

b) for $2.433<a<2.979, x^{p}<x^{e}$, and $q^{p}>q^{e}$;

c) for $2.979<a<3.375, x^{p}<x^{e}$, and $q^{p}<q^{e}$;

d) for $2.25<a<3.375, W^{p}>W^{e}$; 
e) $e^{*}$ and $h^{*}$ are decreasing in $a .{ }^{20}$

Proposition 5 demonstrates that, under the present specification, the performance standard leads to both a higher $R \& D$ and a higher output when the market size is small (part a), while the opposite holds for the large market size (part c). For the medium market size (part b), the comparison is mixed: the performance standard leads in terms of output but trails in terms of R\&D. As to an intuitive account of the forces at work here, one can recall the direct effect and the strategic effect invoked by Montero (2002) and summarized above.

Specifically, following an increase in market size (from $a=2.3$ to $a=2.5$ and from $a=3.1$ to $a=3.3$ ), which in turn induces an increase in the level of output, the regulator concerned with social welfare maximization reduces the amount of pollution allowed under both policy instruments (as shown in the fifth row in Table 3). Since, under the performance standard, the pollution constraint that maximizes welfare is set as a percentage of output, this constraint is more flexible than the emission standard. Consequently, the final emissions allowed per firm are larger, while the number of units abated per firm becomes lower under the performance standard. In contrast, the production decision of an individual firm does not directly affect the pollution constraint under the emission standard. This intrusive nature of the emission standard enables a firm to generate higher cost savings from abatement (the second row in Table 3 for medium and large market sizes), providing it with more incentives to invest in R\&D. This outcome corresponds to what Montero (2002) refers to as the direct effect. Since the abatement cost is lower under the performance standard, a firm can increase its market share and profit more under this instrument than under the emission standard. Thus, according to Montero (2002), the performance standard is favored for having a stronger strategic effect. The conjunction of these two effects leads to the mixed outcome reported in Proposition 5.

All together then, the comparison between the two instruments in terms of R\&D and output appears as a draw, but the main message of Proposition 5 is that we know the parameter regions for which each instrument is superior for each of two dimensions.

In contrast, we now have the clear-cut outcome that the equilibrium level of welfare remains consistently higher under the performance standard. Postponing a conclusion on the comparison at hand to the end of this section, we note now that the outcome of part $d$ stands in apparent contradiction to the result of part c. Nonetheless, given the many inefficiencies that are usually

\footnotetext{
${ }^{20}$ At $a=3.375, e^{*}=h^{*}=0, x^{e}=x^{p}=c=1, q^{e}=q^{p}=1.125$, and $W^{e}=W^{p}=3.5625$.
} 
associated with strategic behavior, one can reconcile the findings of parts c and d by postulating that the emission standard leads to excessive levels of R\&D and output from a social standpoint. Similar conclusions have appeared in specific instances in the industrial organization literature on strategic R\&D (see e.g., Brander and Spencer, 1983).

The next section analyzes the robustness of the results of Proposition 5 when parameters $a$ and $s$ are held fixed.

\subsubsection{Varying parameter $\gamma$}

This section examines how changes in the cost of $\mathrm{R} \& \mathrm{D}, \gamma$, affect the comparison of $\mathrm{R} \& \mathrm{D}$ investment, output and welfare under the emission and performance standards, for the threestage game with $a$ and $s$ held constant.

Table 4 on the next page illustrates the results. 


\begin{tabular}{lcccccc}
\hline Variable & e.s. & p.s. & e.s. & p.s. & e.s. & p.s. \\
& $(\gamma=1.15)$ & & $(\gamma=1.20)$ & & $(\gamma=1.80)$ & \\
\hline Standard & 0.09 & 0.50 & 0.17 & 0.52 & 0.40 & 0.62 \\
R\&D per firm & 0.81 & 0.43 & 0.65 & 0.39 & 0.19 & 0.21 \\
Individual output & 0.77 & 0.74 & 0.72 & 0.74 & 0.56 & 0.73 \\
Welfare & 1.64 & 1.70 & 1.61 & 1.69 & 1.53 & 1.66 \\
Final emissions per firm & 0.09 & 0.37 & 0.17 & 0.38 & 0.40 & 0.45 \\
Abatement per firm & 0.68 & 0.37 & 0.55 & 0.36 & 0.16 & 0.28 \\
\hline
\end{tabular}

Table 4: Solution to the three-stage game for $b=c=s=1, a=2.5$ and chosen values of $\gamma$.

Table 4 shows that an increase in the cost of $R \& D$ negatively affects $R \& D$ investment, output, emissions abated per firm, and welfare. The rising cost of R\&D discourages firms from producing, because with the lower R\&D investment, the abatement cost is higher. Consequently, the level of output drops. To recover some of the consumer surplus, the regulator increases the allowed level of pollution (measured by the final emissions per firm in the fifth row in Table 4). This, combined with the lower level of production results in lower emissions abated per firm (the sixth row in Table 4). Despite this effort of the regulator, social welfare decreases. The reason is that a higher $\gamma$ not only leads to a higher cost of $\mathrm{R} \& \mathrm{D}$, but also to a higher level of damage, caused by permitting firms to pollute more. Thus, the regulator faces a clear trade-off between increasing the consumer surplus and decreasing the environmental damage to society.

These and further results are summarized in Proposition 6, in which the lower bound of $\mathrm{R} \& \mathrm{D}$ cost, $\gamma=1.11$, guarantees that the emission constraint, $e^{*}$, is non-negative. The values $a=2.5$ and $s=1$ are chosen for illustrative purposes.

Proposition 6 Let $b=c=s=1$ and $a=2.5$. Then

a) for $1.11<\gamma<1.18, x^{e}>x^{p}$, and $q^{e}>q^{p}$;

b) for $1.18<\gamma<1.67, x^{e}>x^{p}$, and $q^{e}<q^{p}$;

c) for $1.67<\gamma, x^{e}<x^{p}$, and $q^{e}<q^{p}$;

d) $W^{p}>W^{e}$ for $\gamma>1.11$;

e) $e^{*}$ and $h^{*}$ are increasing in $\gamma$.

Proposition 6 shows that an increase in the cost of $R \& D$ investment has a more pronounced 
impact on the levels of output and R\&D under the emission standard. Whereas output affects the pollution constraint optimally set by the welfare maximizing regulator under the performance standard, under the emission standard the output decision of a firm has no direct impact on the pollution constraint set by the social planner. Thus, when $R \& D$ becomes more expensive, a firm reduces its output to decrease the cost of abatement under the intrusive emission standard. Under the more flexible performance standard, maintaining a high level of production enables a firm to decrease the abatement cost. Consequently, when the cost of $R \& D$ rises, the levels of output and R\&D investment under the performance standard become higher than under the emission standard.

Proposition 6 also reveals the lack of a systematic result for the comparison of R\&D investment and output under both instruments across the relevant ranges of parameter $\gamma$ (see parts a-c). In close analogy with the previous result, the comparison between the two instruments concludes with a draw. In contrast, an unambiguous welfare result is obtained (part d). The performance standard is superior in generating a higher level of welfare, in complete accordance with the result obtained in the previous section.

To verify this important result, we next consider the impact that a change in the extent of environmental damage, as measured by parameter $s$, has on the welfare comparison for the two policy instruments.

\subsubsection{Varying $s$}

Clearly, the extent of environmental damage plays an important role in the social welfare maximization problem of the regulator. Hence, this section examines how changes in the extent of environmental damage, as measured by parameter $s$, affect the comparison of the equilibrium levels of R\&D investment, output and welfare under the emission and performance standards, for the three-stage game with other parameters held constant.

Table 5 on the next page illustrates the results.

Since society suffers when environmental damage increases (the fourth row in Table 5), the regulator reduces the level of emissions allowed (the fifth row in Table 5). This leads to a higher level of pollution abated (the sixth row in Table 5). By investing more in R\&D, firms are able to reduce the increased abatement cost. 


\begin{tabular}{lcccc}
\hline Variable & $\begin{array}{c}\text { e.s. } \\
(s=1)\end{array}$ & p.s. & e.s. & p.s. \\
& $0.43=2.7)$ & \\
\hline Standard & 0.65 & 0.10 & 0.22 \\
R\&D per firm & 0.14 & 0.21 & 0.45 & 0.43 \\
Individual output & 0.48 & 0.68 & 0.58 & 0.62 \\
Welfare & 1.26 & 1.37 & 1.11 & 1.15 \\
Final emissions per firm & 0.43 & 0.44 & 0.10 & 0.14 \\
Abatement per firm & 0.05 & 0.24 & 0.48 & 0.48 \\
\hline
\end{tabular}

Table 5: Solution to the three-stage game for $b=c=1, \gamma=1.5, a=2.3$ and chosen values of $s$.

An increase in environmental damage clearly affects the level of production under both policy instruments. Interestingly, whereas output increases under the emission standard, output declines under the performance standard. The reason for the latter effect is that damage, which the regulator wants to reduce, depends on the level of production. Thus, to reduce damage under the performance standard, one must decrease output. Unlike under the performance standard, output under the emission standard increases, since output for this instrument does not enter the term capturing the environmental damage. Proposition 7 summarizes these findings for $a=2.3$. The values of parameters $a, b, c$, and $\gamma$ in Proposition 7 have been fixed for the purpose of illustrating the results.

Proposition 7 Let $b=c=1, \gamma=1.5, a=2.3$ and $s>0.957 .{ }^{21}$ Then

a) if $0.957<s<1.282, x^{p}>x^{e}$, otherwise, $x^{e}>x^{p}$;

b) $q^{p}>q^{e}$;

c) $W^{p}>W^{e}$

d) $e^{*}$ and $h^{*}$ are decreasing in $s$.

Proposition 7 shows that our results are economically meaningful for $s>0.957$. For this range of parameter $s$, we obtain two key findings. First, we show that, as in the previous examinations, the equilibrium level of welfare is higher under the performance standard than under the emission standard. Second, our results suggest that output is higher under the performance standard that

\footnotetext{
${ }^{21} s>0.957$ is required to satisfy (A2), given the parameter specification with $a=2.3$.
} 
under the emission standard. By means of Table 6 and Proposition 8, we show that the latter result is robust to our parameter specification, but, unlike the welfare outcome, it does not hold generally.

\begin{tabular}{lcccc}
\hline Variable & e.s. & p.s. & e.s. & p.s. \\
& $(s=0.60)$ & & $(s=1)$ & \\
\hline Standard & 0.46 & 0.79 & 0.11 & 0.31 \\
R\&D per firm & 0.45 & 0.18 & 0.78 & 0.57 \\
Individual output & 0.85 & 0.98 & 0.96 & 0.94 \\
Welfare & 2.84 & 3.05 & 2.80 & 2.84 \\
\hline Final emissions per firm & 0.46 & 0.77 & 0.11 & 0.29 \\
\hline Abatement per firm & 0.39 & 0.21 & 0.85 & 0.65 \\
\hline
\end{tabular}

Table 6: Solution to the three-stage game for $b=c=1, \gamma=1.5, a=3.1$ and chosen values of $s$.

Table 6 demonstrates the effect that an increase in the market size, $a$, from $a=2.3$ (Table 5) to $a=3.1$, has on the equilibrium levels of R\&D investment, output and welfare. Particularly, it indicates that for a large market size, the level of output generated under the performance standard no longer exceeds that under the emission standard. The intuition behind this result has already been given in Section 4.3.1., which investigates how changes in parameter $a$ affect the equilibrium levels of R\&D investment, output, and welfare, when other parameters, including $s$, are held constant. Proposition 8 provides an overview of the key results obtained in this section, assuming that $a=3.1$, while $s$ varies.

Proposition 8 Let $b=c=1, \gamma=1.5, a=3.1$ and $s>0.55 .^{22}$ Then

a) $x^{e}>x^{p}$

b) if $0.55<s<0.79, q^{p}>q^{e}$ and $q^{p}<q^{e}$ otherwise;

c) $W^{p}>W^{e}$;

d) $e^{*}$ and $h^{*}$ are decreasing in $s$.

Proposition 8 validates the previous results we have obtained regarding the equilibrium welfare comparison between the two policy instruments, according to which, the performance standard generates a higher level of social welfare.

\footnotetext{
${ }^{22} s>0.55$ is required to satisfy (A2), given the parameter specification, with $a=3.1$.
} 
Although a complete analytical comparison that would generalize the four Propositions (Propositions 5-8) to all relevant parameter regions does not appear tractable, we managed to confirm this result via thorough numerical simulations covering the entire parameter space.

Since welfare is the most natural indicator on which to base the comparison for social and regulatory purposes, we conclude on the basis of Propositions 5, 6, 7, and 8 that the performance standard is the superior regulatory instrument of the two. Indeed, as argued above, the absence of a clear-cut comparison for R\&D and output levels is a secondary consideration.

\section{Conclusion}

This paper has extended the analysis of environmental R\&D incentives in Cournot markets by Montero (2002) in two key respects. First, we analyze the incentives to R\&D provided by two command-and-control instruments, emission and performance standards, by focusing on the standard linear specification of Cournot oligopoly. For Montero's two-stage game, this enables us to derive many relevant full-fledged equilibrium properties, including a complete characterization of the parameter regions for which each standard is superior according to the $R \& D$ and industry output criteria, respectively. We also establish the abscence of a clearcut conclusion for the comparison of the two policy instruments in terms of social welfare, by providing specific examples showing that the comparison can go either way. Thus, for the two stage games associated with the two command-and-control instruments, we provide a complete characterization of the comparative performance of the two instruments.

In an attempt to address an important and natural issue not considered by Montero (2002), the second objective of this paper is to endogenize the regulatory standard for the two games analysed in this paper. To do so, for each of the two instruments, we consider a three-stage game with the planner maximizing social welfare in an initial stage, followed by the previous two-stage game. This extended game leads to an unambiguous result that equilibrium welfare is higher under the socially optimal performance standard. Thus, with endogenous regulatory targets, we obtain the clear-cut conclusion that the performance standard is welfare superior to the emission standard.

Contrasting the two different types of analysis conducted here and their associated conclusions, we confirm once again the importance of integrating welfare analysis into the study of pollution regulation in imperfectly competitive markets. Indeed, a clear-cut conclusion that 
would hold uniformly for all feasible regulatory targets is thus perhaps simply too much to expect. Instead, it is certainly of interest to learn that a performance standard is the way to go for the most relevant target, naturally defined as the socially optimal level.

\section{Appendix}

This section contains the proofs and computations for all the results of the paper.

Proof of Lemma 1. In the last stage, firm $i$ solves the optimization problem (2), with first order condition (or FOC)

$$
-2 b q_{i}+a-b q_{j}-c+x_{i}=0
$$

The reaction function of firm $i$ is given by

$$
q_{i}\left(q_{j}, x_{i}\right)= \begin{cases}\frac{a-c+x_{i}}{2 b}-\frac{q_{j}}{2}, & \text { if } \frac{a-c+x_{i}}{b} \geq q_{j} \\ 0, & \text { otherwise }\end{cases}
$$

The objective function is strictly concave, thus, a unique maximum is attained (the secondorder condition $(\mathrm{SOC})$ is $-2 b<0)$. Solving simultaneously the two non-zero reaction functions of the firms yields (for $i \neq j$ )

$$
q_{i}\left(x_{i}, x_{j}\right)=\frac{a-c+2 x_{i}-x_{j}}{3 b}
$$

In the first stage, firm $i$ chooses the level of R\&D, $x_{i}$, given $x_{j}, e$ and $q_{i}\left(x_{i}, x_{j}\right)$ in equation (9). The FOC of (3) is then given by

$$
\frac{2}{3 b}\left[-2 b q_{i}\left(x_{i}, x_{j}\right)+a-b q_{j}\left(x_{i}, x_{j}\right)-c+x_{i}\right]+\frac{4}{3} q_{i}\left(x_{i}, x_{j}\right)-e-\gamma x_{i}=0 .
$$

The first term in (10) vanishes by the FOC in (8). By (A1), $9 b \gamma>8$, the SOC holds. Plugging equation (9) into equation (10) and using the fact that the firms are symmetric and subject to the same standard $e$, we get that every firm invests

$$
x(e)=\frac{4(a-c)-9 b e}{9 b \gamma-4}
$$

in $\mathrm{R} \& \mathrm{D}$, yielding an output of

$$
q(e)=\frac{3 \gamma(a-c)-3 e}{9 b \gamma-4}
$$


Since $e$ is selected exogenously, we want to choose values that are economically meaningful. In particular, we want $0<x(e)<c$ and $0<e<q(e)$; we show that $0<e<\frac{3 \gamma(a-c)}{9 b \gamma-1}$ is sufficient to guarantee that these two inequalities hold.

First notice that $e$, such that $0<e<\frac{3 \gamma(a-c)}{9 b \gamma-1}$, implies that $\frac{(a-c)}{9 b \gamma-1}<x(e)=\frac{4(a-c)-9 b e}{9 b \gamma-4}<\frac{4(a-c)}{9 b \gamma-4}$ by multiplying the first inequality by $-9 b$, adding $4(a-c)$ and dividing by $9 b \gamma-4$, which is positive by (A1). Assumption (A1) implies that the left hand side of the second inequality is strictly positive, hence, $0<x(e)$. The proof of $x(e)<c$ also follows from (A1); to see it rewrite $9 b \gamma c>4 a$ as $9 b \gamma c-4 c>4(a-c)$, which implies that $\frac{4(a-c)}{9 b \gamma-4}<c$, hence, that $x(e)<c$.

To show that $e<q(e)$ (which immediately implies that $0<q(e)$ ) consider the following implications:

$e<\frac{3 \gamma(a-c)}{9 b \gamma-1}$, hence, $(9 b \gamma-1) e<3 \gamma(a-c)$ by (A1) and, further, $9 b \gamma e-4 e+3 e<3 \gamma(a-c)$, thus, $(9 b \gamma-4) e<3 \gamma(a-c)-3 e$, consequently leading to $e<\frac{3 \gamma(a-c)-3 e}{9 b \gamma-4}=q(e)$. This completes the proof of Lemma 1 .

Proof of Lemma 2. In the last stage, firms solve problem (4), with FOC:

$$
a-2 b q_{i}-b q_{j}-\left(c-x_{i}\right)(1-h)=0
$$

with SOC $-2 b<0$.

Solving simultaneously for the positive parts of the reaction curves of the firms, gives us

$$
q_{i}\left(x_{i}, x_{j}, h\right)=\frac{\left(2 x_{i}-x_{j}-c\right)(1-h)+a}{3 b} .
$$

Using equation (14) in problem (5) helps derive the following FOC for the first stage:

$$
\frac{2}{3 b}(1-h)\left[a-2 b q_{i}\left(x_{i}, x_{j}, h\right)-b q_{j}\left(x_{i}, x_{j}, h\right)-\left(c-x_{i}\right)(1-h)\right]+\frac{4}{3} q_{i}\left(x_{i}, x_{j}, h\right)(1-h)-\gamma x_{i}=0,
$$

with SOC $8(1-h)^{2} /(9 b)-\gamma<0$, which holds by (A1), $8<9 b \gamma$, and $0<h<1$.

The first term in equation (15) vanishes by FOC (13). By the symmetry of the model, equations (14) and (15) imply our result, $x(h)=\frac{4(1-h)[c(1-h)-a]}{4(1-h)^{2}-9 b \gamma}$ and $q(h)=\frac{3 \gamma[c(1-h)-a]}{4(1-h)^{2}-9 b \gamma}$.

Note that the following assumption: (A') $8(1-h)^{2}<9 b \gamma, a>c(1-h)$ and $9 b c \gamma>4 a(1-h)$ for all $0<h<1$, guarantees that $0<x(h)<c$ and $q(h)>0$. It is easy to verify that $0<h<1$ and (A1) $(8<9 b \gamma, a>c$ and $9 b c \gamma>4 a)$ imply $\left(\mathrm{A}^{\prime}\right)$, since the lowest side of the (positive) inequalities is multiplied by 
a number between 0 and 1 . This completes the proof of Lemma 2 .

Proof of Proposition 3. To avoid confusion between the equilibrium variables, we add the superscript $e$ for the equilibrium under the emission standard, and $p$ for the equilibrium under the performance standard. Thus, $x^{e}(e)$ and $q^{e}(e)$ correspond to $x(e)$ and $q(e)$ in Proposition 1, respectively; and $x^{p}(h)$ and $q^{p}(h)$ correspond to $x(h)$ and $q(h)$ in Proposition 2 , respectively.

Now, let us fix $e=h q^{p}(h)$, so that both instruments generate the same level of emissions. Hence, $e$ is a function of $h$ and we can write $x^{e}$ in terms of $h, x^{e}(e)=x^{e}\left(h q^{p}(h)\right)=x^{e}(h)$. Similarly, we can write $q^{e}$ as a function of $h$ (this feature will be used in part c).

For parts a and b, we find the values of $h$ for which $x^{e}(h)=x^{p}(h)$ holds. For these values, we can analyze the functions of interest to see which function takes a larger value on given intervals. It turns out that there are only two solutions: $h_{1}=0$, which is trivial and implies zero level of emissions, and $h_{a b}=\frac{(45 c-9 a) b \gamma-16 a}{9 b c \gamma-16 a}$, which is the threshold of interest. Since there are only two roots, we analyze the slopes of $x^{e}(h)$ and $x^{p}(h)$ around zero, to prove our result. We have to distinguish between two cases, which depend on the sign of $9 b c \gamma-16 a$.

a) Suppose that $9 b c \gamma-16 a>0$. Two possibilities arise; either $h_{a b}>0$ or $h_{a b}<0$. First, let us assume that $h_{a b}>0$. Since, by assumption, its denominator is positive, the numerator must be positive as well, i.e., $(45 c-9 a) b \gamma-16 a>0$. Further, it can be shown that $x^{e \prime}(0)-x^{p \prime}(0)=$ $-\frac{(45 c-9 a) b \gamma-16 a}{(4-9 b \gamma)^{2}}$ is negative by the statement above, thus, $x^{p}(h)$ is above $x^{e}(h)$ for all $0<h<h_{a b}$. A similar argument shows that $x^{e \prime}(0)-x^{p^{\prime}}(0)>0$ when $h_{a b}<0$, which implies that $x^{e}(h)>x^{p}(h)$ for all $0<h<1$. To complete our result, it suffices to show that $x^{e \prime}\left(h_{a b}\right)-x^{p^{\prime}}\left(h_{a b}\right) \neq 0$, which holds by our assumptions, since $x^{e^{\prime}}\left(h_{a b}\right)-x^{p^{\prime}}\left(h_{a b}\right)=\frac{((45 c-9 a) b \gamma-16 a)(16 a-9 b c \gamma)^{2}}{9 b \gamma(-64+9 b \gamma)(-4+9 b \gamma)\left(-4 a^{2}+9 b c^{2} \gamma\right)}$.

b) Now suppose that $9 b c \gamma-16 a<0$. The proof of this part is analogous to that in part a, and is therefore omitted.

c) This proof follows the same strategy as the one implemented in parts a and b. We look for $h$ that satisfies the equality $q^{e}(e)=q^{p}(h)$, with $e=h q^{p}(h)$. Again two solutions are obtained; the trivial root $h_{1}=0$, and the root of interest, $h_{c}=\frac{9 b c \gamma+c-5 a}{c-4 a}$. Note that (A1) implies that $h_{c}<1$, yet, it remains to establish whether its value is positive or negative. First, let us suppose that $h_{c}>0$ and analyze the slopes of $q^{e}(h)$ and $q^{p}(h)$ at $h_{1}=0$. If $h_{c}>0$, then $9 b c \gamma+c-5 a<0$, by (A1), $a>c$. The reader can verify that further rearrangments lead to $q^{e \prime}(0)-q^{h^{\prime}}(0)=-\frac{3 \gamma(9 b c \gamma+c-5 a)}{(4-9 b \gamma)^{2}}$, which is positive by the assumption $h_{c}>0$ and $\gamma>0$. 
Hence, $q^{e}(h)$ is always above $q^{h}(h)$ (for all $0<h<h_{c}$ ). On the other hand, if $h_{c}<0$, then $9 b c \gamma+c-5 a>0$ and $q^{e \prime}(0)-q^{h^{\prime}}(0)<0$; thus, $q^{h}(h)$ is above $q^{e}(h)$ for all $0<h<1$. The fact that $q^{e \prime}\left(h_{c}\right)-q^{h^{\prime}}\left(h_{c}\right)=-\frac{3(-4 a+c)^{2} \gamma(9 b c g+c-5 a)}{(-4+9 b \gamma)(-1+36 b \gamma)\left(-4 a^{2}+9 b c^{2} \gamma\right)}$ differs from zero (given our assumptions in any case), completes our proof.

Proof of Lemma 4. Given the results in Lemma 1, we solve the problem of the regulator presented in equation (6). Here, the FOC is given by the following equation:

$$
\frac{d q(e)}{d e}[a-2 b q(e)-c+x(e)]+\frac{d x(e)}{d e}[q(e)-e-\gamma x(e)]+c-x(e)-2 s e=0 .
$$

The SOC of this problem is $\frac{2(9 b+8 s-18 b \gamma s)}{9 b \gamma-4}$, which is negative by (A1) and (A2). Using the FOCs (8) and (10), equation (16) becomes

$$
b q(e) \frac{d q(e)}{d e}-\frac{1}{3} q(e) \frac{d x(e)}{d e}+c-x(e)-2 s e=0 .
$$

Combining equations (11), (12) and (17), leads to the results outlined in Lemma 4. (A1) and (A2) guarantee that in equilibrium, $0<x^{*}<c$ and $0<e^{*}<q^{*}$. 


\section{References}

Amir, R., 2000, Modelling imperfectly appropriable R\&D via spillovers. International Journal of Industrial Organization 18, 1013-1032.

Amir, R., M. Germain, V. Van Steenberghe, 2008, On the impact of innovation on the marginal abatement cost curve, J. Public Econ. Theory, 10, 985-1010.

Baker, E., L. Clarke, E. Shittu, 2008, Technical change and the marginal cost of abatement, Energy Econ., 30, 2799-2816.

Bauman, Y., M. Lee, K. Seeley, 2008, Does technological innovation really reduce marginal abatement costs? Some theory, algebraic evidence, and policy implications, Environ. Resour. Econ., 40, 507-527.

Bréchet, T., P. Jouvet, 2008, Environmental innovation and the cost of pollution abatement revisited, Ecol. Econ., 65, 262-265.

Benchekroun, H., and N. Van Long, 1998, Efficiency inducing taxation for polluting oligopolists, Journal of Public Economics, 70, 325-342.

Brander, J., and B. Spencer, 1983, Strategic commitment with R\&D: The symmetric case, Bell Journal of Economics, 14, 225-235.

Bruneau, J. F., 2004, A note on permits, standards, and technological innovation, Journal of Environmental Economics and Management, 48, 1192-1199.

D'Aspremont, C., and A. Jacquemin, 1988, Cooperative and noncooperative R\&D in duopoly with spillovers, The American Economic Review, 78, 1133-1137.

Downing, P. B., and L. W. White, 1986, Innovation in pollution control, Journal of Environmental Economics and Management, 13, 18-29.

Harrington, W., Morgenstern, R., and T. Sterner, 2004, Choosing environmental policy: comparing instruments and outcomes in the United States and Europe, RFF Press.

Hueth, B., and T. Melkonyan, 2009, Standards and the regulation of environmental risk, Journal of Regulatory Economics, 36, 219-246. 
Jung, C., Krutilla, K., and R. Boyd, 1996, Incentives for advanced pollution abatement technology at the industry level: An evaluation of policy alternatives, Journal of Environmental Economics and Management, 30, 95-111.

Malueg, D. A., 1989, Emission credit trading and the incentive to adopt new pollution abatement technology, Journal of Environmental Economics and Management, 16, 52-57.

Milliman, S. R., and R. Prince, 1989, Firms incentives to promote technological change in pollution control, Journal of Environmental Economics and Management, 17, 247-265 (1989).

Montero, J. P., 2002, Permits, Standards, and Technology Innovation, Journal of Environmental Economics and Management, 44, 23-44.

Requate, T., 2005, Dynamic incentives by environmental policy instruments - survey, Ecological Economics, 54, 175-195.

Viscusi, W. K., Vernon, J. M., and J. E. Harrington, 2000, Economics of regulation and antitrust, MIT Press. 